\title{
Epidemiología del paciente gran quemado adulto en Chile: experiencia del Servicio de Quemados del Hospital de la Asistencia Pública de Santiago
}

\author{
CLAUDIA R. ALBORNOZ, JORGE VILLEGAS, \\ VERÓNICA PEÑA, SANDRA WHITTLE
}

\section{Sociodemographic/clinical characteristics and outcomes of patients admitted to the National Burn Center of Chile}

Background: Approximately 150 subjects per year suffer severe burns in Chile. Aim: To analyze sociodemographic/clinical features and outcomes of severely burned patients. Material and Methods: Retrospective cohort study of 936 patients aged $47 \pm 20$ years ( $66 \%$ males), admitted to the National Burn Center of Chile between 2006 and 2010. Sociodemographic/clinical and burn variables and outcomes were studied. Results: Mean total percentage of body surface area burned was $27 \pm 20 \%$. A quarter of the patients had social features that could jeopardize rehabilitation. Fire was the burning agent in 73\%, which along with electricity presented greater lethality $(p<0.01)$. Inhalation injury was diagnosed in $22 \%$ of the patients. Twenty eight percent of patients had impaired consciousness at the moment of the accident, leading to larger burns, higher incidence of inhalation injury and greater lethality. Lethality for severe, critical and exceptional survival groups was 8.4, 37.7 and 70.4\%, respectively. Conclusions: Severely burned patients in Chile are mainly males at working age. Fire is the main agent and $28 \%$ had impaired consciousness, which was associated with an increase in the severity of burns. Knowledge of the characteristics and outcomes of the patients is important to implement prevention and treatment strategies adjusted to the national reality.

(Rev Med Chile 2013; 141: 181-186).

Key words: Burns (MeSH); Economics; Epidemiology (MeSH).

L as quemaduras son un problema de salud pública a nivel mundial tanto por su mortalidad como por su morbilidad, que se traduce en discapacidad. Según datos de Organización Mundial de la Salud en 2002 hubo 320.000 muertes por quemaduras en el mundo ${ }^{1}$. De acuerdo a la Asociación Americana de Quemaduras, que lleva el registro de 70 centros de quemados de Estados Unidos de Norteamérica en su base de datos, entre el 2000 y 2010 hubo 148.000 egresos hospitalarios por quemaduras, 5.540 muertes y un gasto aproxi-

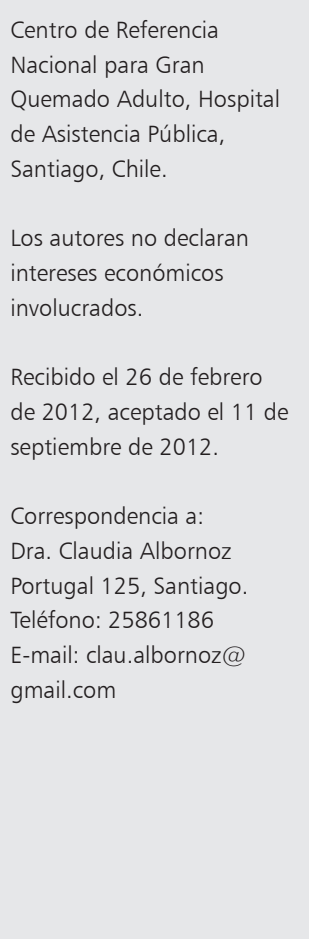

mado por tratamiento de pacientes quemados de 1.000 millones de dólares al año ${ }^{2}$.

En Chile, anualmente más de 6.000 pacientes requieren hospitalización por quemaduras; el año 2007 el Ministerio de Salud (MINSAL) registró 6.435 egresos hospitalarios por quemaduras y el Instituto Nacional de Estadísticas informó 569 muertes por esta causa. La tasa de mortalidad específica por quemaduras en Chile fue 4,5 por 100.000 habitantes para ese año. En el último estudio de carga de enfermedad del MINSAL del 
2004 se estimó que las quemaduras producían la pérdida de 7.891 años de vida ajustados por discapacidad (AVISA) ${ }^{3}$.

Los avances en el tratamiento han determinado una tendencia nacional a la disminución de la mortalidad en todos los grupos etáreos entre los años 1969 y 2000, salvo en el grupo de adultos mayores ${ }^{4}$. En nuestro país no existen datos acerca de las tasas de reinserción social ni laboral, tampoco acerca de las oportunidades ni costos de rehabilitación.

Anualmente aproximadamente 200 pacientes se queman gravemente por causas no laborales y logran recibir atención médica. De éstos, alrededor de $90 \%$ son referidos y atendidos en el Centro de Referencia Nacional del Gran Quemado adulto del Hospital de Asistencia Pública Alejandro del Río (Posta Central). Este es el único centro para pacientes beneficiarios del sistema público de salud (correspondiente al 75\% de la población chilena) para el tratamiento de pacientes quemados graves. Recibe pacientes derivados de todo el país, que cumplen con los criterios clínicos de "gran quemado" establecidos en las guías Clínicas del Programa de Garantías Explícitas en salud (GES): pacientes con Índice de gravedad de Garcés (IG) > 70 puntos o con quemaduras de espesor intermedio $(A B)$ o profundas (B) de $>20 \%$ de superficie corporal quemada (SCQ), pacientes > 65 años con $10 \%$ o más de quemadura $\mathrm{AB}$ o $\mathrm{B}$, pacientes con quemaduras respiratorias o por inhalación de humo, pacientes con quemaduras eléctricas por alta tensión, pacientes quemados politraumatizados y/o con patologías graves asociadas.

El objetivo de este trabajo es analizar los aspectos epidemiológicos y desenlaces de los pacientes quemados graves ingresados al Centro de Referencia Nacional de Chile.

\section{Material y Métodos}

Análisis descriptivo de la cohorte histórica de pacientes ingresados a la Unidad de Cuidados Intensivos e intermedios del Servicio de Quemados del Centro Nacional de Referencia Chileno entre enero de 2006 y diciembre de $2010(n=936)$. La fuente de información fue la base de datos prospectiva del Servicio de Quemados, complementada con la base de datos del Hospital de Asistencia Pública y revisión de registros clínicos en casos necesarios.
Se registraron variables sociodemográficas (edad, género), nivel socioeconómico (definido operacionalmente como bajo y medio beneficiarios de Fonasa, alto afiliados a ISAPRES), de la quemadura (superficie corporal quemada, agente productor de la quemadura, lugar/circunstancias, antecedentes de factores que alteran la conciencia), y desenlaces (incidencia de injuria inhalatoria, letalidad, estadía hospitalaria). También se consignaron limitantes sociales para rehabilitación, tales como ceguera, daño orgánico cerebral, etc.

Se consideró que el paciente presentaba compromiso de conciencia en el momento de la quemadura si se consignaba crisis epiléptica, ebriedad, intoxicación por drogas o compromiso cualitativo de conciencia (pacientes bonzo).

Se analizó número de ingresos, letalidad y estadía hospitalaria por año y grupo de gravedad de Garcés y se comparó con datos históricos del Servicio desde el año 2000 al $2005^{5}$.

Las diferencias entre variables categóricas se analizaron con $\chi^{2}$ o test de Fisher, según correspondiera. La edad y superficie corporal quemada se analizó con test no paramétrico de Mann-Whitney. La dosis letal 50, que es un indicador usado en quemados que establece la superficie corporal con la que fallece el 50\% de los pacientes, se obtuvo mediante regresión logística. Los análisis estadísticos se realizaron con el programa Stata ${ }^{\circledR}$ versión 11.0 (Stata Corporation, College Station, Texas).

\section{Resultados}

Entre 2006 y 2010 ingresaron 936 pacientes al Centro de Referencia Nacional. La edad promedio fue de 47,2 años ( $\pm 20,1$, rango 15-97 años). La distribución de pacientes por edad se presenta en la Figura 1. Se observó que la gran mayoría de los pacientes se concentra en el grupo de menores de 60 años (72\%); sin embargo, 260 pacientes (28\%) eran mayores de 60 años. Aproximadamente 10\% de los pacientes presentaron una edad mayor o igual a 80 años.

Los ingresos por quemaduras graves son más frecuentes en el género masculino, 65,7\% comparado con $34,3 \%$ en el género femenino. El $99 \%$ de los pacientes pertenecía al nivel socioeconómico medio-bajo. Veinticuatro por ciento de los pacientes presentaban alguna característica social que potencialmente dificultaba la rehabilitación 


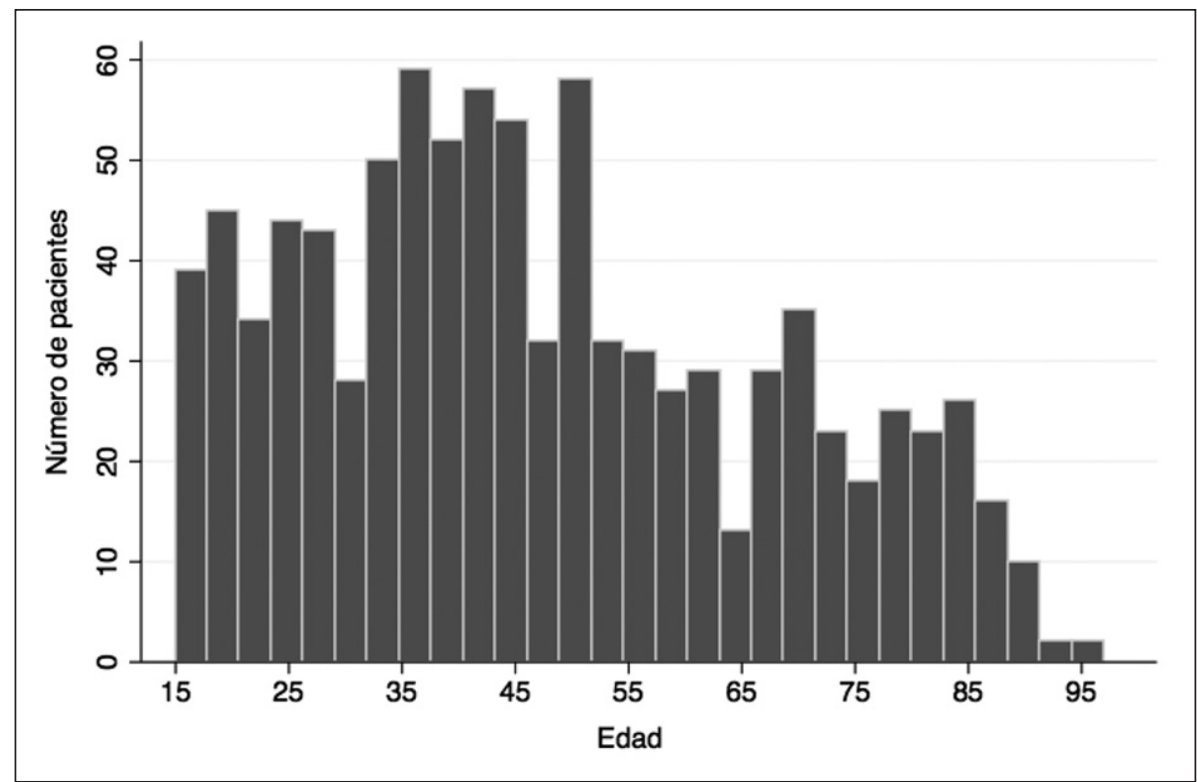

Figura 1. Distribución por edad de pacientes ingresados 2006-2010. Centro de Referencia Nacional del gran quemado adulto, HUAP. (abandono social, drogadicción, alcoholismo, pacientes privados de libertad, deterioro orgánico cerebral, antecedentes psiquiátricos, secuela neurológica o depresión).

La superficie corporal quemada media fue $27 \%$ ( $\pm 20 \%$, rango $1-98 \%$ ), con superficie quemada profunda al ingreso en promedio 8,5\% $( \pm 13 \%)$. La dosis letal 50 fue de $43 \%$, significando que con esa superficie corporal fallece el $50 \%$ de los pacientes.

El agente productor de la quemadura más frecuente fue el fuego en $73 \%$ de los casos, seguido por escaldaduras (18\%) y electricidad (8\%). Las quemaduras químicas y por contacto son excepcionales en este grupo de pacientes, correspondiendo a $2 \%$ en total (Figura 2). La letalidad según agente es significativamente distinta $(p<0,01)$, siendo mayor para los pacientes con quemaduras por fuego y eléctricas (Tabla 1).

La Tabla 2 muestra el lugar/circunstancia del accidente: el sitio más frecuente de ocurrencia de la quemadura fue el domicilio, seguido por accidentes laborales y en la vía pública. La quemadura fue resultado de una agresión en 31 pacientes (3,3\%) y autoagresión (quemadura a lo bonzo) en 86 pacientes $(9,2 \%)$.

Se encontró compromiso de conciencia en 261 pacientes $(28 \%)$ en el momento de la quemadura y éstos pacientes presentaron significativamente una mayor letalidad (36\% comparado con $26 \%$, $p<0,01)$. Los pacientes con compromiso de conciencia presentan además superficies corporales quemadas más extensas ( $29 \%$ comparado con $22 \%, \mathrm{p}<0,01)$. Injuria inhalatoria fue diagnosticada en $22 \%$ de los pacientes y fue significativamente más frecuente en los pacientes que presentaron

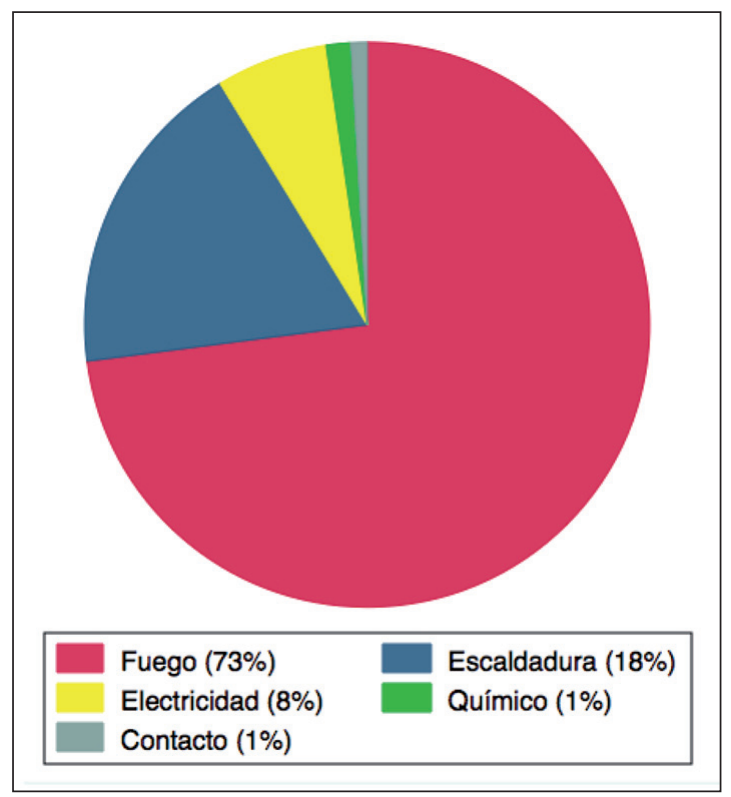

Figura 2. Agente productor de la quemadura. 
compromiso de conciencia al momento de la quemadura ( $48 \%$ versus $22 \%, \mathrm{p}<0,01$ ).

Los pacientes con quemaduras autoinferidas (a lo bonzo) constituyen un subgrupo especial de pacientes. La mayoría son de género masculino (70\%). La prevalencia de enfermedades psiquiátricas es mayor en este grupo, $17 \%$ comparado con $2 \%(\mathrm{p}<0,01)$, presentan significativamente mayor injuria inhalatoria que en los pacientes no autoinferidos (59\% comparado con $18 \%, \mathrm{p}<0,01)$ y mayores superficies corporales quemadas promedio (39\% comparado con $22 \%, \mathrm{p}<0,01)$. La letalidad en el grupo de los pacientes bonzo es mayor que en los pacientes no autoinferidos, $30 \%$

Tabla 1. Letalidad según agente productor de la quemadura

\begin{tabular}{|lcc|}
\hline Agente & Total de pacientes & \% fallecidos \\
\hline Fuego & 626 & 23,8 \\
\hline Escaldadura & 157 & 7,0 \\
\hline Electricidad & 55 & 9,0 \\
Químico & 12 & 8,3 \\
\hline
\end{tabular}

Tabla 2. Lugar o circunstancia del accidente

\begin{tabular}{|lcc|}
\hline Lugar/circunstancia & n & \% \\
\hline Domicilio & 637 & 68,0 \\
\hline Trabajo & 86 & 9,2 \\
Vía pública & 33 & 3,5 \\
Agresión & 31 & 3,3 \\
Autoagresión & 86 & 9,2 \\
Desconocidas & 63 & 6,8 \\
\hline
\end{tabular}

comparado con $18 \%(p<0,01)$. Sin embargo, este efecto desaparece al ajustar por injuria inhalatoria y superficie corporal quemada.

Aproximadamente el $60 \%$ de los pacientes ingresados fue categorizado como grave, crítico o de sobrevida excepcional. El número de pacientes ingresados y la letalidad por grupo de gravedad se muestra en la Tabla 3. La letalidad en el grupo grave fue de $8,4 \%$, aumentando a $37,7 \%$ y $70,4 \%$ en los grupos críticos y de sobrevida excepcional, respectivamente.

Sin embargo, en los últimos 10 años se ha observado una tendencia a la disminución de la letalidad en todos los grupos, especialmente en el grupo crítico en que se observa una disminución de la letalidad de $70 \%$ a $31 \%$ y en el grupo de sobrevida excepcional de $93 \%$ a $77 \%$ entre los años 2000 y 2010 (Figura 3).

La estadía hospitalaria es mayor en los grupos grave y crítico ( 35,4 y 48,7 días respectivamente), disminuyendo en el grupo de sobrevida excepcional debido a la alta letalidad.

\section{Discusión}

Los pacientes quemados graves, ingresados al Centro de Referencia Nacional (HUAP) son en su mayoría pacientes en edad laboralmente activa. Se queman mayoritariamente por fuego y un cuarto de los pacientes presenta algún factor de oscurecimiento de conciencia que condiciona la quemadura. El análisis de las características epidemiológicas de los pacientes quemados adultos en Chile permite establecer al menos tres grupos objetivo de prevención que deberían abstenerse de manipular fuego: personas bajo efectos del alcohol y pacientes epilépticos por el compromiso de con-

Tabla 3. Número de Ingresos, letalidad y estadía hospitalaria por grupo de gravedad de Garcés 2006-2010

\begin{tabular}{lccc}
\hline Grupo de gravedad de Garcés & $\begin{array}{c}\text { Número de ingresos } \\
\mathbf{n}(\%)\end{array}$ & $\begin{array}{c}\text { Letalidad por grupo } \\
\text { (\%) }\end{array}$ & $\begin{array}{c}\text { Estadía hospitalaria } \\
\text { (días, ds) }\end{array}$ \\
\hline Leve (IG 21-40) & $115(12,3)$ & 0,8 & $9,2( \pm 8,2)$ \\
\hline Moderado (IG 41-70) & $266(28,4)$ & 1,1 & $20,5( \pm 17,7)$ \\
\hline Grave (IG 71-100) & $265(28,3)$ & 8,4 & $35,4( \pm 28,4)$ \\
\hline Crítico (IG 101-105) & $167(17,9)$ & 37,7 & $48,7( \pm 38,2)$ \\
\hline Sobrevida Excepcional (IG $\geq 151)$ & $123(13,1)$ & 70,5 & $33,6( \pm 30,2)$ \\
\hline
\end{tabular}

IG: Índice de gravedad, ds: desviación estándar. 


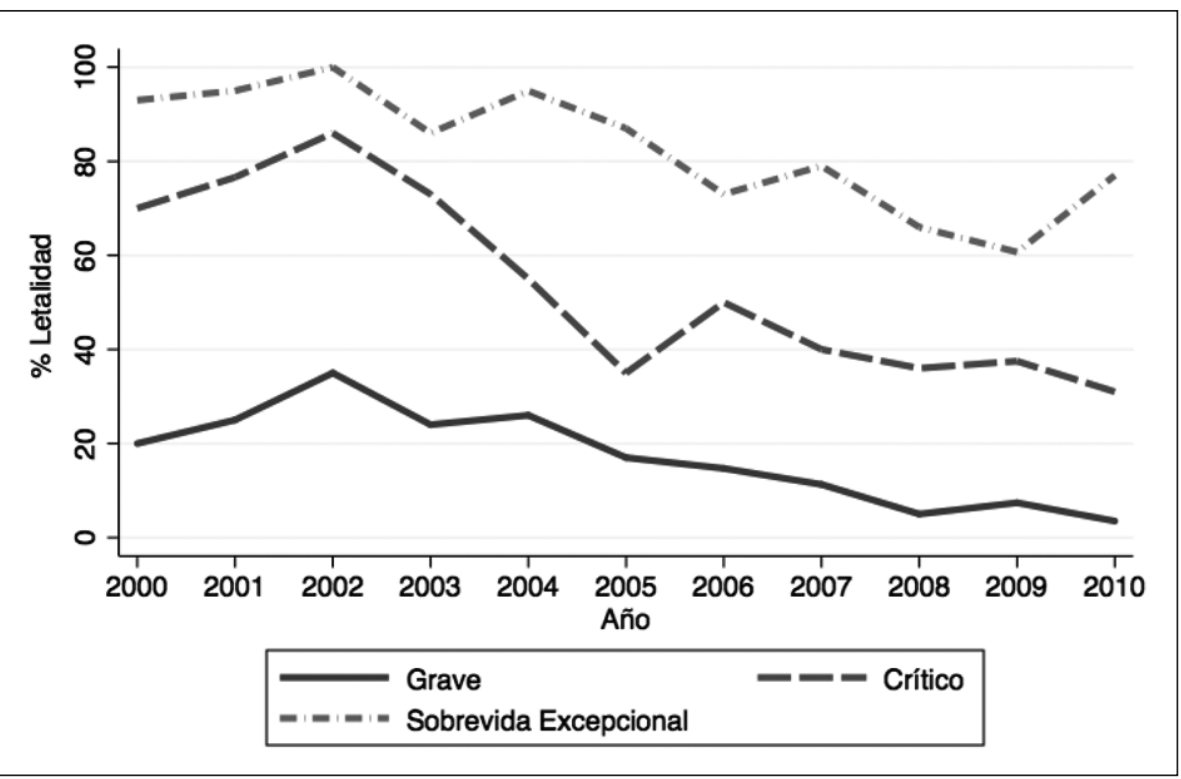

Figura 3. Tendencias de la letalidad por grupo de gravedad de Garcés 2000-2010. Centro de Referencia Nacional del gran quemado adulto (HUAP). ciencia asociado, y pacientes añosos por razones que se explican más adelante.

Los pacientes con compromiso de conciencia presentan mayor letalidad, mayor injuria inhalatoria y superficies corporales quemadas más extensas, ya que niveles disminuidos de conciencia no permiten una adecuada reacción ante la noxa que es la quemadura. Un grupo especial de alteración de conciencia son los pacientes con quemaduras autoinferidas o bonzo, que presentan compromiso cualitativo de conciencia. Estos pacientes intencionadamente no evitan el daño, condicionando quemaduras más extensas y mayor injuria inhalatoria. En un estudio cualitativo realizado en nuestro centro, se encontró que estos pacientes frecuentemente presentan rasgos de personalidad limítrofes, trastornos afectivos y adictivos y generalmente no existe intencionalidad suicida en el momento de autoinferirse la quemadura, es más bien una respuesta impulsiva frente a alguna situación (datos no publicados).

Las tasas de mortalidad específicas por quemaduras en nuestro país han demostrado una disminución en los últimos 50 años, salvo en el grupo de adultos mayores ${ }^{4}$. En la medida que se produzca la transición epidemiológica proyectada para Chile, se espera un aumento de adultos mayores quemados sobre la proporción actual de $28 \%{ }^{5}$. Los pacientes añosos constituyen un grupo especialmente vulnerable, debido a que se ha observado que la mortalidad triplica la de los pacientes más jóvenes con la misma superficie corporal quemada. Existe un porcentaje de pacientes añosos en los que se logra una adecuada cobertura cutánea, pero que debido a su reserva funcional disminuida y condiciones médicas asociadas no logran sobrevivir ${ }^{6,7}$.

En nuestro país, en los últimos años se han impulsado políticas de Estado para garantizar acceso y equidad en la atención de los pacientes quemados graves:

- La intervención del protocolo quirúrgico en el Servicio de Quemados del Hospital de Asistencia Pública Alejandro del Río del año 2004 produjo una disminución de la letalidad de 19\%, llegando a cifras de letalidad global de $37 \%{ }^{8}$.

- La inclusión del tratamiento del gran quemado en el Programa de Garantías Específicas (GES) y la publicación de guías de tratamiento el año $2007^{5}$.

- La creación de un Centro de Referencia Nacional con Unidad de Cuidados intensivos exclusiva para pacientes quemados en el Hospital de Asistencia Pública de Santiago (HUAP) el año 2008.

Todos estos cambios, han permitido la entrega de un tratamiento oportuno, estandarizado y de 
calidad sin discriminar por edad, porcentaje de superficie corporal quemada, patologías agregadas, procedencia ni nivel socioeconómico para pacientes beneficiarios del sistema público de salud. Significó además oportunidad de tratamiento para pacientes que antes se consideraban fuera de alcance terapéutico (grupo de sobrevida excepcional, índice de gravedad $>150$ ). Este grupo de pacientes previo al año 2004 no eran considerados una prioridad de traslado, por lo que el tratamiento se realizaba en el lugar de origen con la limitación de recursos que eso supone. Entre el 2006 y el 2010 se han tratado 123 pacientes del grupo de sobrevida excepcional, logrando sobrevida en $30 \%$ de los casos (36 pacientes). Este aumento de la sobrevida en pacientes con quemaduras más extensas, conduce a un aumento en el número de pacientes que requieren terapia de rehabilitación intensiva y cirugía de las secuelas, que muchas veces la red de salud no está en condiciones de afrontar. La discapacidad y reinserción social de los pacientes quemados aún no han sido evaluadas en nuestro país.

Los positivos cambios anteriormente expuestos en cuanto a oportunidad de atención, financiamiento, la creación de guías clínicas y de un centro nacional con un tratamiento estandarizado han llevado a la disminución de la letalidad en todos los grupos en los últimos 10 años lo que sin duda significa un gran avance (Figura 3). Sin embargo, la dosis letal 50 de $43 \%$ de nuestro país está lejos aún de la de países desarrollados como Estados Unidos de Norteamérica en que se encuentra alrededor de $70 \%$ de superficie corporal quemada ${ }^{2,9}$.

Las limitaciones de este estudio son principalmente la inclusión de pacientes tratados exclusivamente en el Centro de Referencia Nacional, no incluyendo pacientes que han debido ser derivados a otros centros por falta de disponibilidad de camas en éste. Tampoco incluye a pacientes con quemaduras producidas en el ambiente laboral "formal" que son tratados en las mutualidades de accidentes laborales con una realidad epidemiológica, económica y de rehabilitación diferentes a la presentada.

Como conclusión, en Chile ha habido grandes avances en los últimos años en el tratamiento de los pacientes quemados ${ }^{5,8}$. Sin embargo, hay desafíos pendientes como seguir mejorando los resultados en sobrevida y la implementación de estrategias de prevención y rehabilitación. Estos temas requieren urgentemente de la atención de los encargados de las políticas públicas de salud.

\section{Referencias}

1. World Health Organization. Fact sheet about burns 2010. http://wwwwhoint/topics/burns/en/, Consultado en noviembre de 2011.

2. American Burn Association. National Burn Repository 2011 Report. Dataset Version 7.0. http://www.ameriburn.org/2011NBRAnnualReport.pdf Consultado en enero de 2012, 2011.

3. Ministerio de Salud de Chile. Informe Final Estudio de carga de enfermedad y carga atribuible 2007. http://epi. minsal.cl/epi/html/invest/cargaenf2008/Informe\%20 final\%20carga_Enf_2007.pdf Consultado en noviembre de 2011.

4. Danilla Enei S, Pasten Rojas J, Fasce Pineda G, Díaz Tapia V, Iruretagoyena Bruce M. Mortality trends from burn injuries in Chile: 1954-1999. Burns 2004; 30: 34856.

5. Ministerio de Salud de Chile. Guía Clínica Gran Quemado 2007. http://www.redsalud.gov.cl/archivos/guiasges/ GPCGes-GranQuemado-2007.pdf Consultada en noviembre de 2011.

6. Albornoz CR, Villegas J, Bravo I, Peña V. El GES promueve la equidad en el tratamiento del gran quemado de la tercera edad. Rev Med Chile 2011; 139: 1465-70.

7. Albornoz CR, Villegas J, Sylvester M, Peña V, Bravo I. Burns are more aggressive in the elderly: proportion of deep burn area/total burn area might have a role in mortality. Burns 2011; 37: 1058-61.

8. Villegas J, Torres E, Pedreros C, Singh P, Longton C, Said $\mathrm{J}$, et al. Mortalidad tras un año de protocolización en el manejo del paciente quemado. Rev Chilena de Cirugía 2010; 62: 144-9.

9. Belgian outcome in Burn injury study group. Development and validation of a model for prediction of mortality in patients with acute burn injury. Br J Surg 2009; 96: 111-7. 